\title{
Stent tunnel technique to save thrombosed native hemodialysis fistula with extensive venous aneurysm
}

\author{
Martin Rabellino' \\ Guillermo J Rosa-Diez ${ }^{2}$ \\ Sergio A Shinzato' \\ Pablo Rodriguez' \\ Oscar A Peralta' \\ Maria S Crucelegui ${ }^{2}$ \\ Rosario Luxardo ${ }^{2}$ \\ Agustina Heredia-Martinez ${ }^{2}$ \\ Mariela I Bedini-Rocca ${ }^{2}$ \\ Ricardo D García-Mónaco' \\ 'Department of Angiography and \\ Endovascular Therapy, ${ }^{2}$ Department \\ of Nephrology, Hospital Italiano de \\ Buenos Aires, Buenos Aires, Argentina
}

Correspondence: Guillermo J Rosa-Diez Department of Nephrology, Hospital Italiano de Buenos Aires, JD Perón 4190, Buenos Aires CI I99ABB, Argentina

Tel +54 9l। 4565 548।

Email Guillermo.rosadiez@

hospitalitaliano.org.ar
This article was published in the following Dove Press journal: International Journal of Nephrology and Renovascular Disease 27 July 2017

Number of times this article has been viewed

Introduction and purpose: The increasing number of patients undergoing hemodialysis and the limited number of access sites have resulted in an increasing number of techniques to maintain vascular access for hemodialysis. Thrombosed arteriovenous (AV) fistulas with large venous aneurysms have poor treatment results, with both endovascular and surgical techniques, leading to a high rate of definitive AV access loss. The purpose of this study was to review the feasibility and initial results of this novel endovascular treatment of thrombosed AV fistulas with large venous aneurysms.

Materials and methods: A novel endovascular treatment technique of inserting nitinol autoexpandable uncovered stents stretching through the whole puncture site area, thus creating a tunnel inside the thrombus, was retrospectively analyzed and described.

Results: A total of 17 stents were placed in 10 hemodialysis fistulas, with a mean venous coverage length of $17.8 \mathrm{~cm}$. In all the cases, $100 \%$ technical success was achieved, with complete restoration of blood flow in all patients. There were no procedure-related complications. The mean follow-up was 167 days (range 60-420 days), with a primary and assisted patency of $80 \%$ and $100 \%$, respectively. No multiple trans-stent struts-related complications were observed. Three stent fractures were diagnosed with plain films at the site of puncture without consequence in the venous access permeability.

Conclusion: The "stent tunnel technique" is a feasible, safe and effective alternative to salvage native hemodialysis access, thus extending the function of the venous access with no signs of stent-related complications and a respectable midterm patency.

Keywords: vascular access, hemodialysis, endovascular procedure, thrombosed native hemodialysis, aneurysm, stent, stent tunnel technique

\section{Introduction}

Dysfunction of vascular hemodialysis access in patients with end-stage renal disease is a major health problem associated with increased morbidity, mortality and health care costs. Therapeutic alternatives in nonfunctioning or thrombosed vascular access include open surgical repair and various endovascular techniques, such as percutaneous transluminal angioplasty (PTA), thrombectomy, thromboaspiration and fibrinolytic drug treatment. Underlying lesions can also be treated using balloon catheters alone or in combination with intravascular stents. If, after these efforts, reflow is not achieved, the vascular access gets abandoned. ${ }^{1}$ A precipitating factor for acute thrombosis of a native hemodialysis fistula is the presence of venous aneurysms. Recently, self-expandable covered stents have been used for the treatment of thrombosed and non-thrombosed pseudoaneurysms in hemodialysis fistulas with good initial clinical results. ${ }^{2-5}$ With 
that experience as a background, we developed the "stent tunnel technique" which is based on the placement of a self-expanding non-covered nitinol stents inside the thrombi covering the whole length of the puncture site to reestablish the blood flow.

The objective of this retrospective study was to review the feasibility and initial results of a novel endovascular treatment of thrombosed arteriovenous (AV) fistulas with large venous aneurysms.

\section{Materials and methods}

A total of 10 patients with native fistulas in which flow restoration could not be achieved through conventional percutaneous techniques were treated using the stent tunnel technique. All patients were informed about the procedure and signed the corresponding informed consent. The research protocol ethics committee of our hospital approved the collection of data from patients' clinical records, preserving the confidentiality of their identity.

\section{Angiographic technique}

The concept of this technique consists of placing uncovered self-expanding nitinol stents from the anastomosis to the venous segment distal to aneurysms without thrombus, generating a tunnel or flow channel through the thrombus so that it can later be used to puncture. Figure 1 shows the angiographic technique.

Diagnostic angiography was performed through a brachial artery access (abbocath $20 \mathrm{G}$ ), as arterial access can be of great help during recanalization, since it allows proper control of the physiological (antegrade) flow. Intravenous bolus of $5000 \mathrm{U}$ of heparin was administered, while antibiotic prophylaxis was not deemed necessary.

In the first five patients a manual thromboaspiration with catheter was initially attempted, but failed. Therefore, in the same procedure, the stent tunnel technique was performed with good results.

In the remaining five patients, the stent tunnel technique was performed directly.

In the patients who attempted thromboaspiration, the stents were inserted through the same vascular access. In the latter group of patients, the stents were inserted through the femoral vein $(n=1)$, internal jugular vein $(n=2)$ and the vein distal to the anastomosis $(n=2)$. In all patients, a $0.89 \mathrm{~mm}$ hydrophilic guidewire was used to perform the recanalization of access.

The stents were inserted using primary stent technique (no previous dilation) with the objective of covering the whole thrombosed segment and dialysis puncture sites.

After stent placement, PTA was performed using balloon catheters according to the stent diameters. The mean of stent diameter used was $7-8 \mathrm{~mm}$ and was determined by the diameter of the anastomosis. Finally, the sheaths were removed from the vein puncture site suturing the vascular access and applying local compression to the corresponding femoral or jugular vein. In the arterial puncture site, local compression was manually applied for 5 minutes. Procedure was considered successful when residual stenosis was $<30 \%$
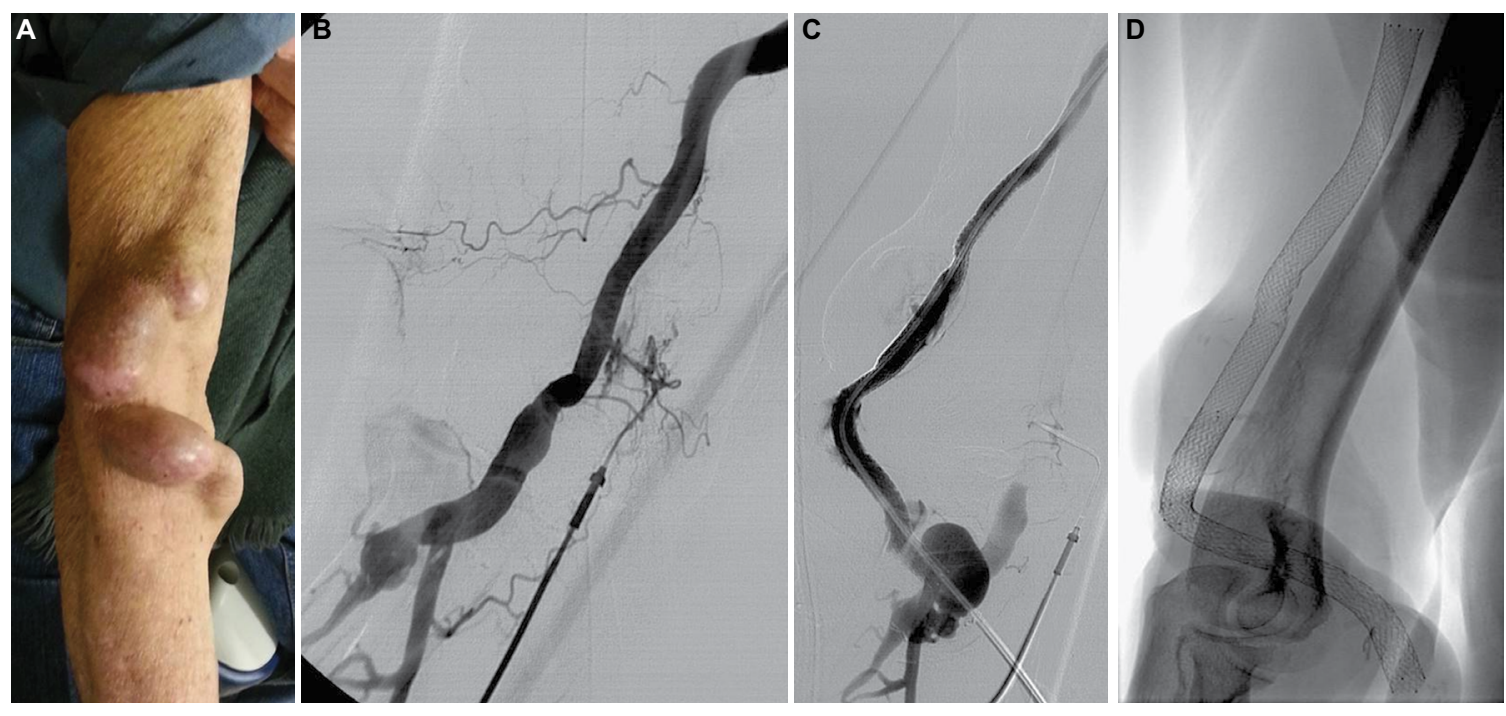

Figure I Showing the angiographic technique in patient case 9.

Notes: (A) Photograph of the URL showing a native humerocephalic fistula with large venous aneurysms on the puncture site area. (B) Angiography of the URL obtained through an abbocath catheter that shows vascular access thrombosis. (C) Control fistulogram showing patency of the whole vascular access with contrast material flowing inside the stents through an intrathrombal channel: stent tunnel technique. (D) Radiography of the stents during follow-up.

Abbreviation: URL, upper right limb. 
while achieving complete flow restoration and hemodialysis could be performed through the AV fistula.

Lifelong antiaggregation with $100 \mathrm{mg}$ acetylsalicylic acid was indicated.

Follow-up was performed using Doppler ultrasound and plain films to evaluate vascular patency and stent integrity, respectively.

\section{Results}

All treated AV accesses were thrombosed native fistulae with one or more venous aneurysms. All patients were undergoing chronic hemodialysis. Eight were male and two were female. The mean age was 76 years old (range 54-87 years old).

All patients were treated within the first week of thrombosed vascular access. The characteristics of the treated hemodialysis fistulas and the stents used are shown in Table 1. Primary patency was $80 \%$ while assisted primary patency was $100 \%$. All treated fistulas presented with large venous aneurysms. In nine cases, the aneurysms were located at the arm while in the remaining fistula the aneurysm was found at the forearm.

A total of 20 self-expandable non-covered nitinol stents were placed for hemodialysis access salvage, on average two stents per fistula. The mean stent-covered length was $17 \mathrm{~cm}$ (range 6-26 cm).

Restoration of blood flow was achieved in all cases. No procedure-related complications were observed up to 30 days after treatment. Eight of the vascular accesses were successfully used for hemodialysis the day after stent placement, while in two patients the vascular access was successfully used 1 week after treatment.

There were no puncture site-related complications (16 $\mathrm{G}$ needle) during hemodialysis. The mean follow-up was 167 days (range 60-420 days). During the follow-up, two patients died from non-procedure-related deaths. One of the patients suffered from an abdominal aortic aneurysm rupture, while the second one died of sudden death at home. In both cases, hemodialysis was still being performed through the stented AV fistula. In the remaining eight patients, two needed a second intervention due to high venous pressure during hemodialysis, one after 70 days and another one after 182 days. In the first patient, contrast medium injection showed a stenosis of the distal cephalic vein. This lesion was not observed in the initial angiography and was therefore considered "de novo." Dilation of the stenosis was performed with an $8 \mathrm{~mm}$ high-pressure balloon catheter (Extreme; Cordis, Miami Lakes, FL, USA). In the second patient, stent fracture in one of the puncture sites was observed and multiple areas of stenosis were found during diagnostic angiography. High-pressure balloon angioplasty of the stenotic areas was performed (Extreme) while an additional stent was placed on the fracture site. Both patients were able to continue using the $\mathrm{AV}$ fistulas for hemodialysis without any further complication.

During the follow-up, three patients suffered from stent fractures at the puncture site without impact on vascular patency nor did it result in other complications. Nevertheless, it was decided that fracture sites should be avoided when puncturing the fistulae.

In three patients, follow-up Doppler ultrasound showed recanalization of venous aneurysms, evidenced by flow around the stents, allowing nurses to puncture these patients avoiding the stents.

\section{Discussion}

Hemodialysis fistulas using a vein conduit have shown superior patency rates than prosthetic grafts. However, in case of thrombosis, venous fistulas are more difficult to treat and patency at short-term follow-up is unsatisfactory. ${ }^{6,7}$

Open surgical repair or endovascular treatment has been proposed. As recommended by the clinical practice guidelines of the National Kidney Foundation (NKF)-Kidney Disease Outcomes Quality Initiative (KDOQI), each institution

Table I Characteristics of the treated hemodialysis fistulas and the stents used

\begin{tabular}{|c|c|c|c|c|c|c|c|c|c|c|}
\hline Patient & $\mathbf{I}$ & 2 & 3 & 4 & 5 & 6 & 7 & 8 & 9 & 10 \\
\hline Fistula location & RUABC & RUABC & RUABC & RUABC & LFRC & LUABC & LUABC & LUABC & RUABC & RUABC \\
\hline Time (months)* & 20 & 40 & 28 & 31 & 110 & 19 & 81 & 108 & 64 & 7 \\
\hline Previous interventions & 2 & 4 & 2 & 3 & 0 & 0 & 2 & 2 & 2 & 0 \\
\hline Aneurysm & One & One & Multiple & Multiple & Multiple & Multiple & Multiple & Multiple & Multiple & Multiple \\
\hline Type of stent & Smart $^{\mathrm{a}}$ & Smart ${ }^{\mathrm{a}}$ & Edwards ${ }^{b}$ & Absolute $^{c}$ & $\begin{array}{l}\text { Absolute } \\
\text { and Smart }\end{array}$ & $\begin{array}{l}\text { Misago }^{\mathrm{d}} \\
\text { and Smart }\end{array}$ & $\begin{array}{l}\text { Smart }{ }^{\mathrm{a}} \text { and } \\
\text { Maris Plus }\end{array}$ & Maris Plus ${ }^{e}$ & Smart ${ }^{\mathrm{a}}$ & Maris Plus \\
\hline Number of stents & 2 & I & 1 & 2 & 3 & 4 & 2 & 2 & 2 & 1 \\
\hline Total length of stent $(\mathrm{cm})$ & 13 & 6 & 17 & 18 & 18 & 26 & 25 & 20 & 22 & 15 \\
\hline
\end{tabular}

Notes: *Time from fistula creation to stent placement. ${ }^{a}$ Cordis, Miami Lakes, FL, USA. 'Edwards Lifesciences Corporation, Irvine, CA, USA. 'Abbott Vascular, Santa Clara, CA, USA. 'Terumo Medical Corp., Somerset, NJ, USA, eMedtronic Invatec, Minneapolis, MN, USA.

Abbreviations: LFRC, left forearm radiocephalic; LUABC, left upper arm brachiocephalic; RUABC, right upper arm brachiocephalic. 
should use their preferred procedures due to the complexity of revascularization techniques. ${ }^{1}$

Endovascular therapies include mechanical thrombectomy, manual catheter aspiration, fibrinolysis and/or removal of the blood clot using a balloon catheter. These techniques can be followed by PTA and stent placement of underlying lesions. No matter which technique is selected to treat this vascular condition; they all need to be assessed with regard to technical success and patency rates. With regard to technical success, the reported result for the previously mentioned techniques ranges from $76 \%$ to $100 \%,{ }^{6}$ while our success rate with the stent tunnel technique was $100 \%$. Our elevated success rate might be explained by the simplicity of our solution which does not attempt to extract the thrombus, an extremely complex task in native thrombosed vascular accesses which are also associated with big venous aneurysm, but rather tries to create a flux tunnel through the insertion of the stents.

With regard to patency success rates, Jain et $\mathrm{al}^{8}$ reported treating 41 vascular hemodialysis accesses using mechanical thrombectomy resulting in a primary patency of $20 \%$ at 6 months with a secondary patency of $54 \%$. With the use of manual catheter aspiration, 73 hemodialysis accesses were treated by Turmel-Rodrigues et $\mathrm{al}^{7}$ reporting a primary patency rate of $9 \%$ at 12 months when the fistulas were localized at the arm and $49 \%$ when localized at the forearm. Secondary patency rates at 12 months were $50 \%$ and $81 \%$, respectively. When fibrinolysis was the preferred technique, Rajan et $\mathrm{al}^{9}$ reported, in 25 treated fistulas a primary patency rate of $36 \%, 28 \%$ and $24 \%$ at 3,6 and 12 months, respectively. In our series, we had a primary patency of $80 \%$ and a primary assisted patency of $100 \%$ after 167 days of the mean follow-up. Even if we take the four patients with longer follow-up, in them we have 302 days of the mean follow-up with primary and primary assisted patency rate of $75 \%$ and $100 \%$, respectively. The use of non-covered self-expandable stents has been previously reported for the treatment of central venous obstructions, recurrent stenosis and stenosis with recoil after PTA and in venous ruptures. ${ }^{10-12}$ There are many reports with covered stents in aneurysmatic vessels for mural thrombus fixation and to prevent thrombus migration with good initial results..$^{2-5,13}$ Zaleski et al ${ }^{14}$ treated $11 \mathrm{AV}$ grafts using metallic stents for intragraft stenosis. During the follow-up, eight stent fractures were detected due to compression and repeated punctures but without clinical impairment. The authors suggested that self-expandable nitinol stents could be more resistant to compression and repeated punctures than metallic ones. Kinning et $\mathrm{al}^{15}$ reported two cases of stent fracture out of 24 fistulae with pseudoaneurysms treated with nitinol-covered stents.

The reported incidence of stent fractures in nitinol stents is low when stents are placed in the arm veins. Stent fractures appear to be a common problem in repeated punctures of dialysis fistulas, as reported by Rhodes and Silas ${ }^{12}$ and Zaleski et al; ${ }^{14}$ however, nitinol stents might be more resistant to stent fractures than balloon-expandable stents. Vogel and Parise ${ }^{16}$ reported in a series of 60 patients with nonfunctioning AV grafts only one case of stent fracture. In this case, the stent was placed crossing the elbow articulation. Supported by these findings, we decided to place self-expandable nitinol stents in hemodialysis fistulas. Nevertheless, we still found stent fractures in three of our patients (30\%), but as no impact on the use of the fistula was reported despite the presence of the fractures, we believe that it might not be a variable that impacts on the permeability of the access.

There are a number of limitations to our study. The first one is we were able to treat only a small number of patients with this technique as it is being used as a last resource in our institution for the time being. The limited number of patients we were able to treat precludes us from deriving evidenced-based conclusions to the utility of this technique in this clinical scenario. Nonetheless, despite the small number of patients presented in this study, this technique has already been used successfully for other vascular conditions in hemodialysis fistulas supporting the safety of the procedure. Another limitation of our study is the short follow-up of our patients, being 2 months the shortest follow-up and almost 9 months our longest follow-up, thus hindering our ability to predict for how much time will the fistula remain usable through the implementation of this technique. Despite this short follow-up, to be able to salvage a hemodialysis fistula and delay the need for the creation of a new one even for a short amount of time is an effort worth making even if in the long run the stent re-thrombosis happened, which is something that has not yet happened in any of our patients.

\section{Conclusion}

The stent tunnel technique could offer a safe, feasible and effective alternative to salvage thrombosed native hemodialysis access, thus extending the function of the venous access with no signs of stent-related complications and a respectable midterm patency. These results need to be confirmed in larger clinical trials. 


\section{Disclosure}

The authors report no conflicts of interest in this work.

\section{References}

1. Clinical practice guidelines and clinical practice recommendations for vascular access, update 2006. Am J Kidney Dis. 2006;48(suppl 1): S4-S5.

2. Gupta M, Rajan DK, Tan KT, Sniderman KW, Simons ME. Use of expanded polytetrafluoroethylene-covered nitinol stents for the salvage of dysfunctional autogenous hemodialysis fistulas. JVasc Interv Radiol. 2008;19(6):950-954.

3. Vesely TM. Use of stent grafts to repair hemodialysis graft-related pseudoaneurysms. J Vasc Interv Radiol. 2005;16(10):1301-1307.

4. Najibi S, Bush RL, Terramani TT, et al. Covered stent exclusion of dialysis access pseudoaneurysms. J Surg Res. 2002;106(1):15-19.

5. Barshes NR, Annambhotla S, Bechara C, et al. Endovascular repair of hemodialysis graft-related pseudoaneurysm: an alternative treatment strategy in salvaging failing dialysis access. Vasc Endovascular Surg. 2008;42(3):228-234.

6. Turmel-Rodrigues L, Raynaud A, Louail B, Beyssen B, Sapoval M. Manual catheter-directed aspiration and other thrombectomy techniques for declotting native fistulas for hemodialysis. J Vasc Interv Radiol. 2001;12(12):1365-1371.

7. Turmel-Rodrigues L, Pengloan J, Rodrigue H, et al. Treatment of failed native arteriovenous fistulae for hemodialysis by interventional radiology. Kidney Int. 2000;57(3):1124-1140.
8. Jain G, Maya ID, Allon M. Outcomes of percutaneous mechanical thrombectomy of arteriovenous fistulas in hemodialysis patients. Semin Dial. 2008;21(6):581-583.

9. Rajan DK, Clark TW, Simons ME, Kachura JR, Sniderman K. Procedural success and patency after percutaneous treatment of thrombosed autogenous arteriovenous dialysis fistulas. JVasc Interv Radiol. 2002;13(12): 1211-1218.

10. Rasmussen RL, Feldman D, Beathard G, Rubin JE. Indications for stent placement in a dialysis access. Semin Dial. 2008;21(1):83-84.

11. Yevzlin A, Asif A. Stent placement in hemodialysis access: historical lessons, the state of the art and future directions. Clin J Am Soc Nephrol. 2009;4(5):996-1008.

12. Rhodes ES, Silas AM. Dialysis needle puncture of Wallgrafts placed in polytetrafluoroethylene hemodialysis grafts. J Vasc Interv Radiol. 2005;16(8):1129-1134.

13. De Blas M, Merino S, Ortiz F, et al. Treatment of popliteal artery aneurysms with uncovered Wallstents. Cardiovasc Intervent Radiol. 1999;22(4):336-339.

14. Zaleski GX, Funaki B, Rosenblum J, Theoharis J, Leef J. Metallic stents deployed in synthetic arteriovenous hemodialysis grafts. AJR Am J Roentgenol. 2001;176(6):1515-1519.

15. Kinning AJ, Becker RW, Fortin GJ, Molnar RG, Dall'Olmo CA. Endograft salvage of hemodialysis accesses threatened by pseudoaneurysms. J Vasc Surg. 2013;57(1):137-143.

16. Vogel PM, Parise C. Comparison of SMART stent placement for arteriovenous graft salvage versus successful graft PTA. J Vasc Interv Radiol. 2005;16(12):1619-1626.

\section{Publish your work in this journal}

The International Journal of Nephrology and Renovascular Disease is an international, peer-reviewed open access journal focusing on the pathophysiology of the kidney and vascular supply. Epidemiology, screening, diagnosis, and treatment interventions are covered as well as basic science, biochemical and immunological studies. The manuscript management system is completely online and includes a very quick and fair peer-review system, which is all easy to use. Visit http://www dovepress.com/testimonials.php to read real quotes from published authors. 